\title{
Whole genome sequencing of Enterobacter mori, an emerging pathogen of kiwifruit and the potential genetic adaptation to pathogenic lifestyle
}

\author{
Mingyang Zhang ${ }^{1,2 \dagger}$, Yilin Zhang ${ }^{3 \dagger}$, Xue Han ${ }^{3}$, Juan Wang ${ }^{1,2}$, Yu Yang ${ }^{3}$, Biao Ren ${ }^{1,2,4}$, Mian Xia ${ }^{5}$, Gang Li, ${ }^{5,6}$, \\ Rongxiang Fang ${ }^{1,2}$, Hang $\mathrm{He}^{3^{*}}$ and Yantao $\mathrm{Jia}^{1,2^{*}}$
}

\begin{abstract}
Members of the Enterobacter genus are gram-negative bacteria, which are used as plant growth-promoting bacteria, and increasingly recovered from economic plants as emerging pathogens. A new Enterobacter mori strain, designated CX01, was isolated as an emerging bacterial pathogen of a recent outbreak of kiwifruit canker-like disease in China. The main symptoms associated with this syndrome are bleeding cankers on the trunk and branch, and brown leaf spots. The genome sequence of E. mori CX01 was determined as a single chromosome of 4,966,908 bp with 4640 predicted open reading frames (ORFs). To better understand the features of the genus and its potential pathogenic mechanisms, five available Enterobacter genomes were compared and a pan-genome of 4870 COGs with 3158 core COGs were revealed. An important feature of the E. mori CX01 genome is that it lacks a type III secretion system often found in pathogenic bacteria, instead it is equipped with type I, II, and VI secretory systems. Besides, the genes encoding putative virulence effectors, two-component systems, nutrient acquisition systems, proteins involved in phytohormone synthesis, which may contribute to the virulence and adaption to the host plant niches are included. The genome sequence of E. mori CX01 has high similarity with that of E. mori LMG 25,706, though the rearrangements occur throughout two genomes. Further pathogenicity assay showed that both strains can either invade kiwifruit or mulberry, indicating they may have similar host range. Comparison with a closely related isolate enabled us to understand its pathogenesis and ecology.
\end{abstract}

\section{Key points}

A new bacterial pathogen (Enterobacter species) was isolated from diseased kiwifruit plant and characterized by $16 \mathrm{~S}$ rDNA sequence.
The whole genome sequence of the isolate was composed of one circular chromosome. The genome comparison with other Enterobacter strains were performed.

The new bacterial causal agent Enterobacter mori CX01 may evolve new strategies to infect kiwifruit plants.

\section{Introduction}

Enterobacter is a genus of gram-negative, rod-shaped and non-spore-forming bacteria belonging to the family Enterobacteriaceae (Hormaeche and Edwards 1960). Enterobacter bacteria are widely distributed in nature, some species function as plant growth promoting bacteria in agriculture (Majeed et al. 2018), while many of
*Correspondence: hehang@pku.edu.cn; jiayt@im.ac.cn

${ }^{\dagger}$ Mingyang Zhang and Yilin Zhang are co-first authors

1 State Key Laboratory of Plant Genomics, Institute of Microbiology,

Chinese Academy of Sciences, Beijing 100101, China

${ }^{3}$ School of Advanced Agriculture Sciences and School of Life Sciences,

State Key Laboratory of Protein and Plant Gene Research, Peking

University, Beijing 100871, China

Full list of author information is available at the end of the article

\section{Springer Open}

C The Author(s) 2021. Open Access This article is licensed under a Creative Commons Attribution 4.0 International License, which permits use, sharing, adaptation, distribution and reproduction in any medium or format, as long as you give appropriate credit to the original author(s) and the source, provide a link to the Creative Commons licence, and indicate if changes were made. The images or other third party material in this article are included in the article's Creative Commons licence, unless indicated otherwise in a credit line to the material. If material is not included in the article's Creative Commons licence and your intended use is not permitted by statutory regulation or exceeds the permitted use, you will need to obtain permission directly from the copyright holder. To view a copy of this licence, visit http://creativecommons.org/licenses/by/4.0/. 
others are frequently isolated from hospitals and recognized as opportunistic pathogens causing various types of human infections (Mezzatesta et al. 2012). According to information in NCBI database, as of May 2021, 28 Enterobacter whole genome sequences were completed. Only two cases E. mori LMG 25706 and E. ludwigii, are phytopathogenic bacteria which cause diseases on mulberry and onion, respectively. The mulberry leaves infected by E. mori LMG 25706 became withered and dry, and then defoliated, the diseased root xylem was moist and discolored with brown stripes, the phloem decayed at the late stage of infection (Zhu et al. 2011). Enterobacter bulb decay caused by E. ludwigii (previous termed E. cloacae) is characterized by a light brown discoloration of the internal fleshy scales (Schroeder et al. 2010). It's worth noting that E. cloacae and other species of this complex have been showing increasing importance as pathogens on various economic plants (e.g., papaya, cassava, rice and cotton) recently (Cao et al. 2020; Garcia-Gonzalez et al. 2018; Nagrale et al. 2020). However, no Enterobacter species associated with kiwifruit bacterial disease have been reported so far.

The kiwifruit (Actinidia spp.) is a worldwide economically and nutritionally important fruit crop. The kiwifruit industry has been seriously threatened by Pseudomonas syringae pv. actinidiae (Psa) (Donati et al. 2014). Canker disease caused by Psa was first identified and described in Japan in 1984 (Takikawa et al. 1989), and had also been reported to break out in 1989 in Cangxi, Sichuan, China (Wang et al. 1992). It is reported that many isolates of $P$. syringae that are phylogenetically closer related to Psa are nonpathogenic and exist as commensals on plants (Xin et al. 2018). The mechanism studies of $P$. syringae virulence factors and genomic and evolutionary insights have revealed basic features of $P$. syringae as a successful plant pathogen (Xin et al. 2018). Similarly, Enterobacter species commonly occur as pathogenic or nonpathogenic bacteria. Understanding the genetic basis of the pathogenic species by comparison with its closely related nonpathogenic bacteria, will help us elucidate the pathogenesis of these microorganisms.

In this study, we identified a new pathogen termed $E$. mori CX01 strain from diseased kiwifruit plant in Cangxi, Sichuan province of China. To our knowledge, this is the first report that Enterobacter species caused canker-like symptoms such as bleeding of kiwifruit vascular tissues and significant vine death. The whole genome sequence information of E. mori strain CX01 should provide the basis for a better understanding of its genetic background. Bioinformatic analysis results uncovered those diverse pathways may help E. mori perceive and respond to environmental cues, and survive in plant tissues.

\section{Materials and methods}

\section{Bacterial collection and preliminary characterization}

The symptomatic samples of kiwifruit trunks, branches and leaves were collected from five regions in Cangxi, Sichuan province, China. The pith juice was taken and diluted with $1 \mathrm{ml}$ distilled water. Leaves were rinsed with sterile distilled water (SDW), disinfested with $0.3 \%$ sodium hypochlorite and rinsed with SDW four times. Small leaf sections were excised from the edge of the diseased tissue and macerated in SDW for $90 \mathrm{~s}$ in a sterile mortar. The above suspensions were streaked onto King's $\mathrm{B}$ (KB) agar plates and incubated for $24 \mathrm{~h}$ at $28^{\circ} \mathrm{C}$ (King et al. 1954). Then, a single colony was successively restreaked onto a new KB agar plate to obtain a pure colony. Initially, the $16 \mathrm{~S}$ rRNA gene was PCR-amplified with bacterial $16 \mathrm{~S}$ rRNA universal primers $27 \mathrm{~F} 5^{\prime}$-AGAGTT TGATCMTGGCTCAG- $3^{\prime}$ and and 1492R 5'-GGTTAC CTTGTTACGACTT-3', 8F 5'-AGAGTTTGATCCTGG CTCAG-3' and U1492R 5'-CGGTTACCTTGTTACGAC TT-3' (Edwards et al. 1989; Lane 1991) and 16S rRNA specific primer pairs of Em-16 s-F 5'-AGCACGTGTGTA GCCCTACTCGTA-3' and Em-16 s-R 5'-AGAGATCTG GAGGAATACCGGTGGC-3'. The amplified PCR product was ligated into cloning vector pClone007 Simple (TSINGKE Biological Technology) and sequenced, then compared with reference sequences available in the GenBank/EMBL/DDBJ databases using BLAST to present the similarity with other bacterial species. Primer pairs of Em-16 s-F and Em-16 s-R were also used in ampliconsequencing for detection $E$. mori in further collected samples. Primer pairs of Psa-16S-23F 5'-TTTTGCTTT GCACACCCGATTT-3' and Psa-16S-23R 5'-CACGCA CCCTTCAATCAGGATG-3' were used to detect Psa strains.

\section{Pathogenicity tests}

E. mori CX01 (CGMCC 1.19108) and LMG25706 (CGMCC 1.10322) inoculation experiments were conducted either on kiwifruit (cultivar Hayward) or on mulberry (variety Baisang) seedlings to confirm bacteria's pathogenicity. Kiwifruit and mulberry leaves were inoculated by scissor clipping with the bacterial suspensions $\left(10^{9}\right.$ colony-forming units $\left./ \mathrm{ml}, \mathrm{cfu} / \mathrm{ml}\right)$, then transferred in a climatic chamber at $25{ }^{\circ} \mathrm{C}$ to detect the necrotic symptoms around the inoculated wounds 6-9 days after inoculation.

Kiwifruit aseptic seedlings were inoculated by vacuum infiltration with the bacterial suspensions $\left(10^{7} \mathrm{cfu} /\right.$ $\mathrm{ml}$ ), after treatment at $4-10{ }^{\circ} \mathrm{C}$ for 3-4 days, kiwifruit seedlings were transferred to $25{ }^{\circ} \mathrm{C}$ plant incubator. At 7-10 days after inoculation, the seedling leaves were ground up and spread on KB agar plates. 
One- to two-year-old vines of kiwifruit (cv. Hongyang) were inoculated by puncturing methods. Fifteen vine sticks were pricked with a needle through a drop $(200 \mu \mathrm{l})$ of bacterial suspension $\left(10^{9} \mathrm{cfu} / \mathrm{ml}\right)$ placed on them, then incubated under $25^{\circ} \mathrm{C}$ for 7 to 10 days to photograph the symptom.

In all above methods, sterile water-treated samples were taken as negative controls. Koch's postulations were verified by characterizing $E$. mori re-isolated from the inoculated samples with similar symptoms. E. mori (CGMCC 1.10322) was bought from China General Microbiological Culture Collection Center.

\section{Genome sequencing and de novo assembly}

Genomic DNA of E. mori CX01 (CGMCC 1.19108) was extracted from fresh bacterial cultures using a modified CTAB (cetyltrimethyl ammonium bromide) method (Murray and Thompson 1980). For single-molecule realtime (SMRT) sequencing (Pacific Biosciences), $15 \mu \mathrm{g}$ DNA was fragmented by partial digestion with Sau3AI, and the DNA fragments at about $10-50 \mathrm{~kb}$ were selected for the library construction and further sequenced on the PacBio Sequel system (Pacific Biosciences, Menlo Park, CA, USA). At least $5 \mu \mathrm{g}$ DNA was used to construct paired-end libraries (PE $150 \mathrm{bp}$ ) and sequenced on Illumina HISEQ platform. A total of $\sim 1.86$ Gb SMRT subreads and $\sim 485 \mathrm{Mb}$ PE reads were produced.

The genome size was estimated using PE reads by KmerGenie (v1.7048) (Chikhi and Medvedev 2014). PacBio subreads were assembled using Canu (v1.8) (Koren et al. 2017) and MECAT (v1.0) (Xiao et al. 2017) respectively, with default parameters and the estimated genome size of 4,961,053 obtained above. In order to compare two genome drafts, we used nucmer to generate alignments between two assemblies, and the draft assembled by MECAT has better continuity, therefore was selected for further analyses. In order to reduce the base error rate, we used the consensus algorithm Quiver and further polished the assembly with PE reads using Pilon.

\section{Gene prediction and functional annotation}

The RAST server was used to annotate the E. mori CX01 draft genome (Darling et al. 2004). Genome annotation was carried out using the Rapid Annotations using Subsystems Technology (RAST) server with the RASTtk annotation scheme and default parameters
(Brettin et al. 2015). The rRNAs of a new genome are simply found using a BLASTN (McGinnis and Madden 2004) search against this curated set which have phylogenetically diverse set of representative sequences of the $23 \mathrm{~S}$ (currently 81 representatives), 16S (currently 120 representatives) and 5S (currently 292 representatives) rRNAs. RAST use the tRNAscan-SE (Lowe and Eddy 1997) to Calling tRNAs. The complete genome sequence of E. mori CX01 has been deposited to the NCBI database under the GenBank accession number CP055276. The KO numbers of the genes and the KEGG metabolic pathway for comparative genome was annotated by KAAS using blast program and database of Prokaryotes (Kanehisa and Goto 2000; Moriya et al. 2007).

\section{Phylogenomic analysis and genome comparison of Enterobacter strains}

Genome alignment between E. mori CX01 and E. mori LMG 25706 was performed by using Mauve (Darling et al. 2004). In order to determine the evolutionary relationship of E. mori CX01 with other bacteria on the phylogenetic tree, four housekeeping genes (gyrB-rpoBatpd-infb) of 44 strains from Enterobacter genus and other 9 different genera were selected to establish phylogenetic tree by neighbor-joining method using MEGA7 software (Kumar et al. 2016). The gene sequences of the selected bacteria are obtained from the NCBI database. The housekeeping gene sequences of E. mori CX01 were obtained by using known gene sequences as queries to blast their homologue genes in the genome with Blastn (McGinnis and Madden 2004).

Gene family clustering was performed using Orthovenn2 under default parameters (Xu et al. 2019) on protein sequences of Enterobacter mori CX01, Enterobacter mori LMG 25706, Enterobacter cloacae (NC_014121.1), Enterobacter cancerogenus (NZ_CP025225.1) and Enterobacter hormaechei (NZ_CP017179.1). The Orthovenn2 software also provides GO enrichment analysis for specific clusters (Xu et al. 2019).

\section{Results \\ Isolation and characterization of a pathogenic bacterium from kiwifruit plants}

In 2018, a serious bacterial disease was investigated at 50 naturally infected kiwifruit orchards in Cangxi, Sichuan

\footnotetext{
(See figure on next page.)

Fig. 1 Disease symptom of E. mori on plants and its epidemics in kiwifruit orchards of Cangxi. a High disease incidence caused by E. mori led to eradication of whole kiwifruit orchards. $\mathbf{b}$ The canker-like disease symptoms on the trunk with white exudation. $\mathbf{c}$ Reddish-brown discoloration under the bark of the trunk. $\mathbf{d}$ Disease symptom of brown spots on kiwifruit leaf. Disease symptom of kiwifruit (Actinidia deliciosa cv. Hayward) leaf after inoculation of E. mori CX01 (e) or E. mori LMG25706 (f). g The symptoms of E. mori CX01 and LMG25706 inoculated mulberry (Morus alba L.)leaf. $\mathbf{h}$ and I Twigs (Actinidia chinensis Cv. Hongyang) showing brownish necrosis after puncturing inoculation with E. mori CX01 or LMG25706
} 

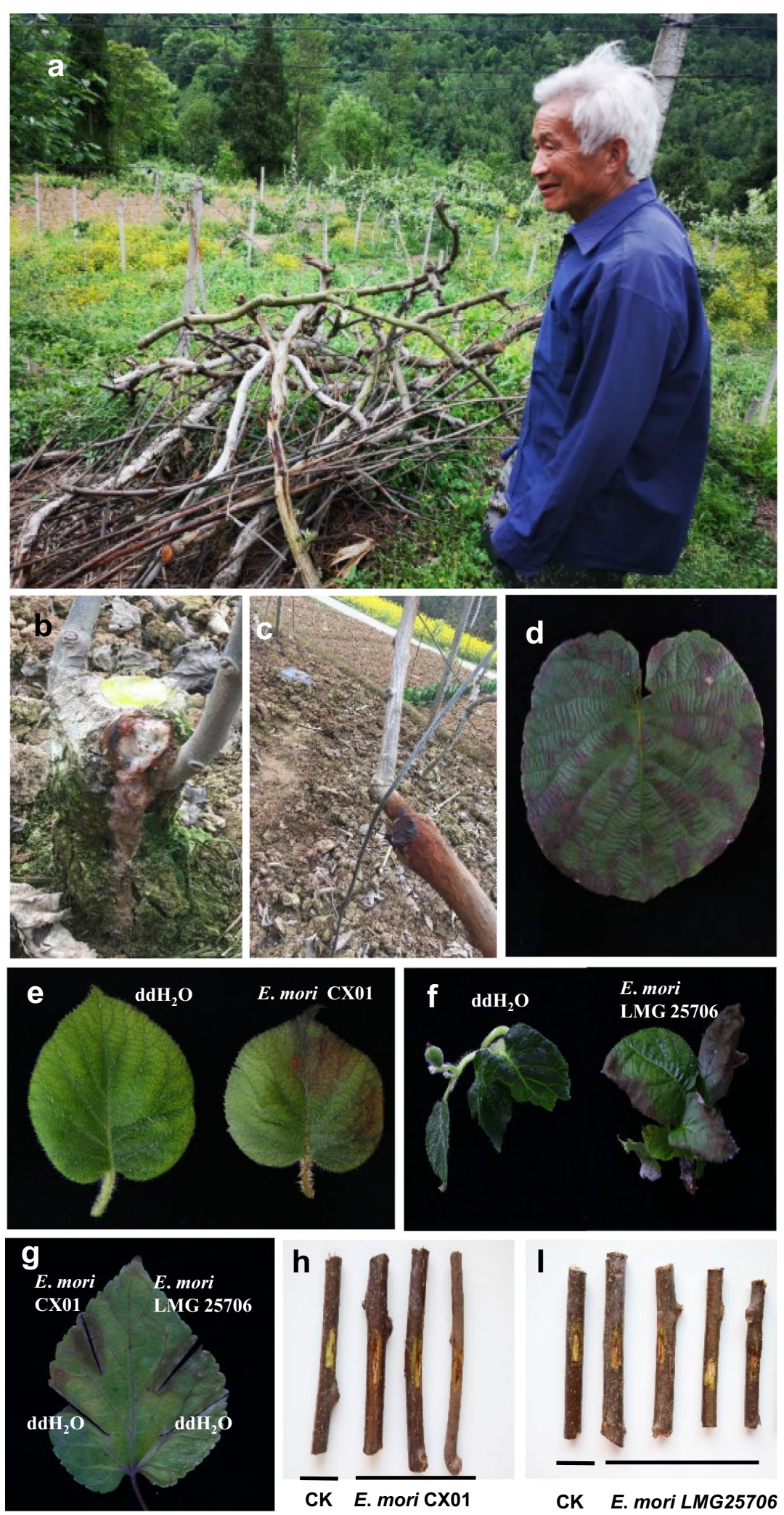

Fig. 1 (See legend on previous page.) 
Province, China. The disease incidence in the field of various cultivars ranged from 50 to $80 \%$, the highest of which was associated with the Actinidia chinensis cultivars, in particular with red-fleshed kiwifruit cultivar 'Hongyang' (always up to 80\%). Wounds and freezing led to more serious symptoms, and eradication of the whole orchard had to be carried out due to the extremely high percentage of disease (Fig. 1a). The main symptoms of the disease were white or brown fluid bleeding cankers in the bark on the trunk and branch during early spring (then termed canker-like disease), brown leaf spots also appeared in summer (Fig. 1b-d), which is similar to the seasonal occurrence rhythm and phenomena of the canker disease caused by Psa (Donati et al. 2014). In severe infections, twig wilting and plant death were observed.

More than 100 bacterial isolates were obtained from 45 diseased trunks and 60 leaves of 1-5-year-old kiwifruit plants, and at least 50 recovered cultures collected from 15 different plants in 5 orchards were analyzed. Surprisingly, no Psa strain was found in these collected tissues. The causal agent of the canker-like disease was identified to belong to the genus Enterobacter based on $16 \mathrm{~S}$ ribosomal RNA (rRNA) gene sequencing performed by using $16 \mathrm{~S}$ rDNA universal primers $(27 \mathrm{~F} / 1492 \mathrm{R}$ and $8 \mathrm{~F} / \mathrm{U1492R}$ ), and further confirmed the molecular characterization of 20 randomly chosen isolates obtained from 8 different plants of 5 orchards by Enterobacter 16S rDNA primer pairs of Em-16s-F and Em-16s-R. The results showed that the bacterial 16S rDNA sequences have the highest identity of $99.2 \%$ with that of $E$. mori LMG 25706 which causes wilt disease on mulberry (Zhu et al. 2011). According to the amended criteria for bacterial species classification, the homology of $16 \mathrm{~S}$ rDNA sequences reaches $98.7-99 \%$ could be classified into the same species (Stackebrandt and Ebers 2006). Then, the new pathogenic bacterium strain was named as $E$. mori CX01. E. mori is gram-negative, facultatively anaerobic, rod-shaped, and motile with polar flagella.

To detect the pathogenicity of E. mori CX01 and E. mori LMG 25706 on kiwifruit, healthy kiwifruit seedlings were inoculated by vacuum infiltration with the suspension of bacterial cells. At least 30 leaves showed brown necrotic phenotype after 7 days of infection by $E$. mori CX01 or E. mori LMG 25706 (Fig. 1e, f). Both E. mori strains could be recovered from the infected leaves. We found that mulberry plants are also susceptible hosts of E. mori CX01 as evaluated by leaf clipping inoculation. At least 10 mulberry leaves were scissor-clipped with bacterial cells of the two strains, dark-brown edges on both sides of wounding leaves were shown after 7 days, whereas water-treatment control remains healthy (Fig. 1g). Since the main symptoms caused by E. mori CX01 was found in vascular tissues, E. mori CX01 and
LMG 25706 were also puncture-inoculated on 15 kiwifruit healthy twigs. Brown lesions were observed at the inoculated sites after 7 days (Fig. 1h, I). The re-isolated bacteria from the diseased tissues were confirmed by $16 \mathrm{~S}$ rRNA gene sequencing. These results fulfilled the Koch's postulates.

\section{Whole-genome sequencing and phylogenetic analysis of $E$. mori}

The genomic DNA of E. mori CX01 was subjected to whole-genome sequencing, which resulted in $1,46,372$ high-quality filtered reads of an average read length of $12,681 \mathrm{bp}$ and coverage equivalent to about 372 times. Quality filtered reads were assembled into scaffolds. The complete genome sequence of E. mori CX01 has been deposited to the NCBI database under the GenBank accession number CP055276. The E. mori CX01 genome has a 4,966,908 base-pair circular chromosome with a $55.40 \% \mathrm{G}+\mathrm{C}$ content. A total of 4640 open reading frames (ORFs) were predicted and annotated. A large proportion of ORFs have been annotated GO (gene ontology) terms. The chromosome has eight $16 \mathrm{~S}$ rRNA genes and 84 tRNAs genes, and 4282 ORFs (92.28\%). The genome reveals the existence of two putative CRISPR (Clustered Regularly Interspaced Short Palindromic Repeat) arrays with the highest evidence level of 4, and no Cas protein detected in the genome by CRISPRCasFinder prediction (Additional file 1: Figure S1).

Phylogenetic analysis was performed by using concatenated nucleotide sequences of housekeeping genes (infB, gyrB, atpD and rpoB) (Fig. 2, Additional file 2: Table S1). MLSA (multilocus sequence analysis) analysis showed that different Enterobacter species clustered together on the phylogenetic tree. The subgroup of $E$. mori species includes E. mori CX01 and seven other strains (the phytopathogens of E. mori LMG 25706, LMG 26284, LMG 26285 and UBA7499, three opportunistic human pathogens of SCEM020047, WCHEM045008 and ECC 1766). The subgroup of $E$. asburiae represented by three clinical strains, which is closely related to $E$. mori subgroup. Other Enterobacter species such as E. cloacae, E. hormaechei, E. cancerogenus and E. ludwigii, most of them were collected from hospital samples, E. cloacae subsp. dissolvens LMG 2683, E. cancerogenus and E. soli were isolated from Zea mays, Populus and Eucalyptus, respectively.

We further performed genome alignment to investigate a collinear relationship among five Enterobacter strains by MAUVE analysis (Fig. 3). Genomic comparison of two phytopathogenic strains (E. mori CX01 and E. mori LMG 25,706) with three clinical isolates, E. cloacae (accession number NC_014121.1), E. cancerogenus (accession number NZ_CP025225.1) and E. hormaechei (accession number NZ_CP017179.1), revealed the 


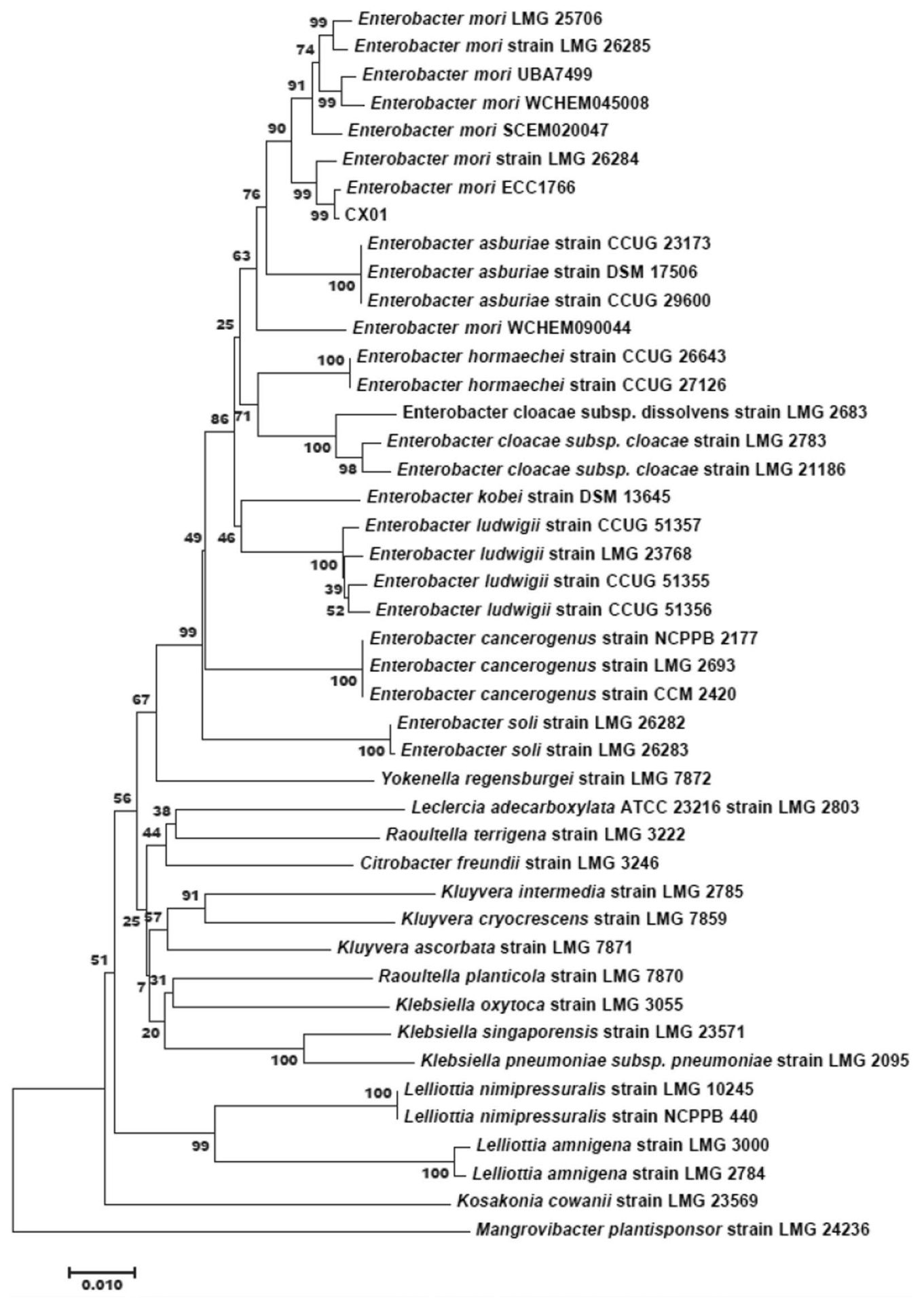

Fig. 2 Phylogenomic analysis of Enterobacter and other genus bacteria. Phylogenetic tree reconstructed from multilocus sequence analysis (MLSA) based on four housekeeping genes gyrB-rpoB-atpD-infB, showing the relationships between E. mori CX01 and the related type strains. The phylogenetic tree was inferred by the neighbor-joining method with the software MEGA7 (Kumar et al. 2016). The bar indicates a $0.01 \%$ nucleotide substitution rate 


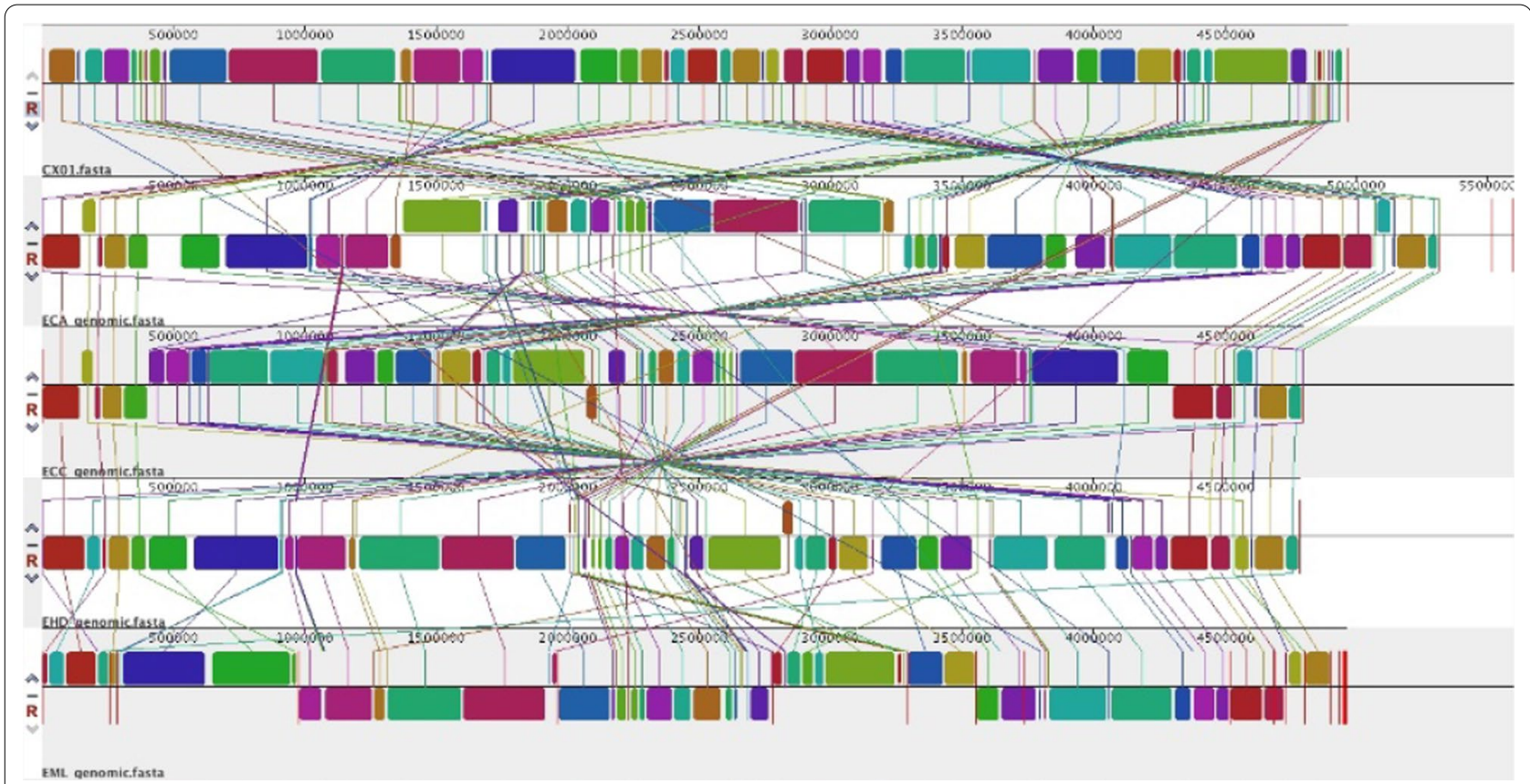

Fig. 3 Genomic alignment of Enterobacter species. Alignment of the genome sequences of E. mori LMG 25706 (EML), E. mori CX01 (CX01, CP055276), E. cloacae ATCC 13047 (ECA, NC_014121.1), E. hormaechei DSM 16691 (EHD, NZ_CP017179.1) and E. cancerogenus CR-Eb1 (ECC, NZ_ CP025225.1) with MAUVE software (Darling et al. 2004). Same color boxes represent homologous regions of the sequences, and are connected by lines

presence of a large core-genome of 3158 COGs (cluster of orthologous groups), and the pan-genome of 4870 COGs as shown in Fig. 4. A set of 111 COGs specifically belongs to E. mori CX01 and LMG 25706, involved in multiple metabolic pathways, including gene expression regulation, biosynthetic and catabolic processes, substrate transport, response to biotic and abiotic stresses and cytolysis process (Additional file 2: Table S2). In E. mori CX01, 224 coding sequences (CDSs) included in the 111 COG classes that arranged in seven fragments, the average GC content of two fragments is $45.7 \%$ and $39.7 \%$, which is much lower than that of the entire genome $(55.40 \%)$, and most likely concerned with horizontal gene transfer events. One fragment (EM_01170135) encodes for AraC family transcriptional regulator, dienelactone hydrolase, superfamily I DNA and RNA helicase, phage integrase, DNA-binding protein, cold shock protein, glucan synthase, Lipid A biosynthesis palmitoleoyltransferase and hypothetical proteins. The other fragment (EM_1323-1329) encodes Rhs-family protein, two tetrapartite efflux system proteins, AraC family transcriptional regulator and hypothetical proteins. In addition, 13 COGs (including $33 \mathrm{CDSs}$ ) from E. mori CX01 were classified as unique, besides hypothetical proteins, 6 GO (gene ontology) terms were represented involved in transposition, hydroxypyruvate reductase activity, isomerase activity, transmembrane transport, phosphoenolpyruvate-dependent sugar phosphotransferase system and sequence-specific DNA binding (Additional file 2: Table S3). While 6 COGs (including $12 \mathrm{CDSs}$ ) were unique to the LMG 25,706 genome (Additional file 2: Table S4) involved in respiratory electron transport chain, protein oxidation, virus tail/fiber and transmembrane transport.

Metabolic pathways shown in subsystem feature categories are conserved among these five Enterobacter species that are characterized by the capability of using diverse carbon sources through multiple carbohydrate metabolic pathways. Over 320 genes were assigned for the role of carbohydrate utilization, accounting for about 7\% of the genes in E. mori CX01 and LMG 25706 (Fig. 5) (Additional file 2: Table S5), and more than 510 genes were significantly enriched in protein metabolism, amino acids and derivatives pathways accounting for $11-13 \%$ of the gene numbers of the two genomes (Additional file 2: Table S6).

\section{Genomic analysis reveals that E. mori CX01 is likely to be better equipped for growth in kiwifruit}

Type III secretion system (T3SS) and Type IV secretion system (T4SS) were absent in E. mori CX01. Other secretion systems including type I, II, and VI secretion systems appeared in the genome, which are believed to be also related to the bacterial virulence (Green and 


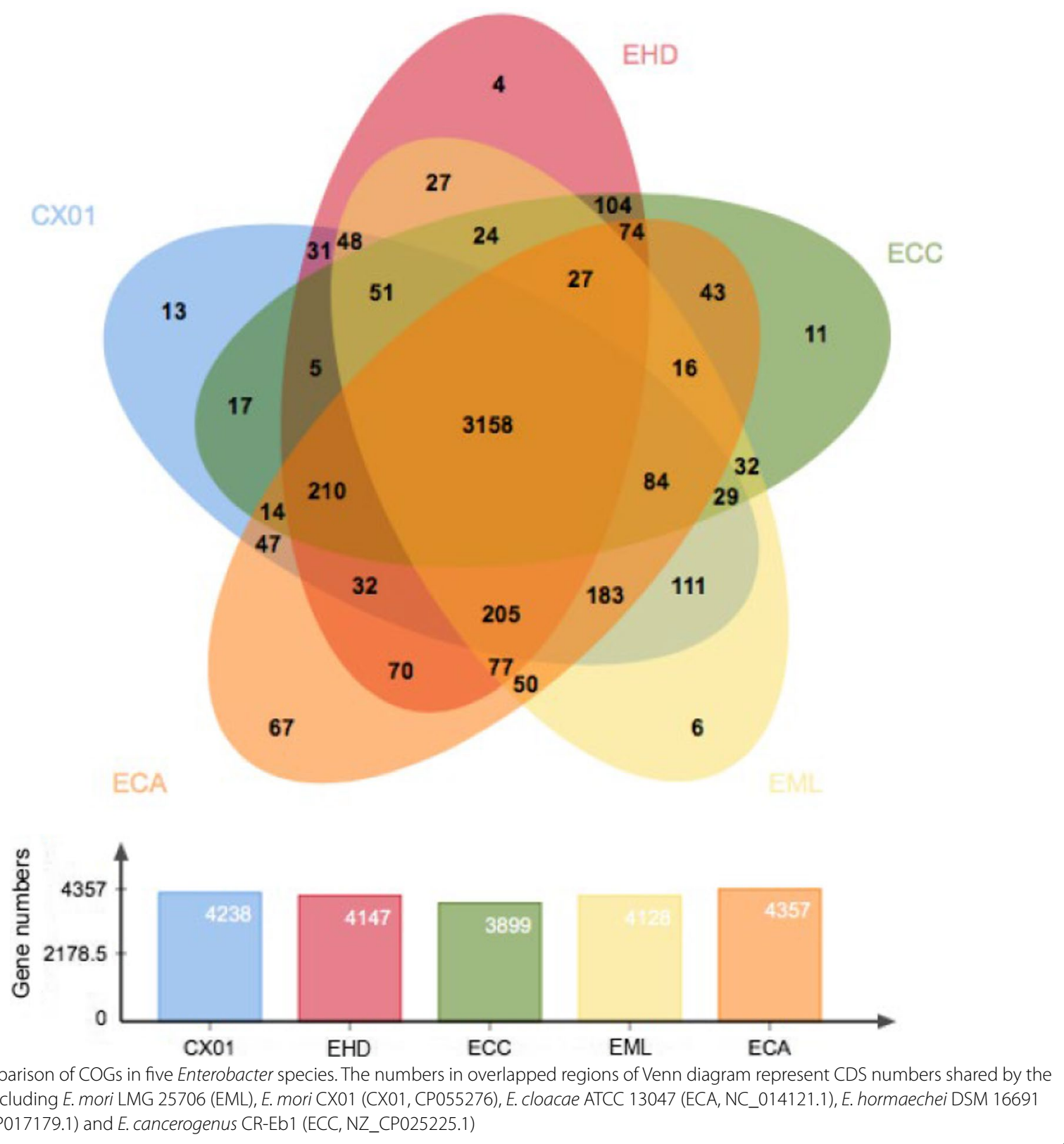

Mecsas 2016). E. mori CX01 has integrate type I secretion system (T1SS) which is conserved in plant and animal bacterial pathogens. Small molecules such as antibiotics and toxins, proteases and lipases, adhesins, and proteins with repeats-in-toxins (RTX) motifs can be exported by T1SS (Green and Mecsas 2016). Type II secretion system (T2SS) that transports folded proteins from the periplasm into the extracellular environment, is important for both pathogenic and non-pathogenic bacteria (Green and Mecsas 2016). In some bacteria, T2SS is still functional in absence of important T2SS genes (de
Vrind et al. 2003). Similarly, E. mori CX01 has incomplete T2SS core genes ( $g s p L K J H G F D E$ ), whether E. mori CX01 has a functional T2SS remains elusive. Type VI secretion system (T6SS) commonly translocates proteins into other bacteria cells, as well as eukaryotic cell targets (Green and Mecsas 2016). T6SSs are hypothesized to contribute to the virulence of some bacterial pathogens, by delivering protein effectors into host cells, and secreting substrates into neighboring bacteria that benefit for interbacterial competing in a specific host niche (Green and Mecsas 2016). The homologues of Hcp (hemolysin 


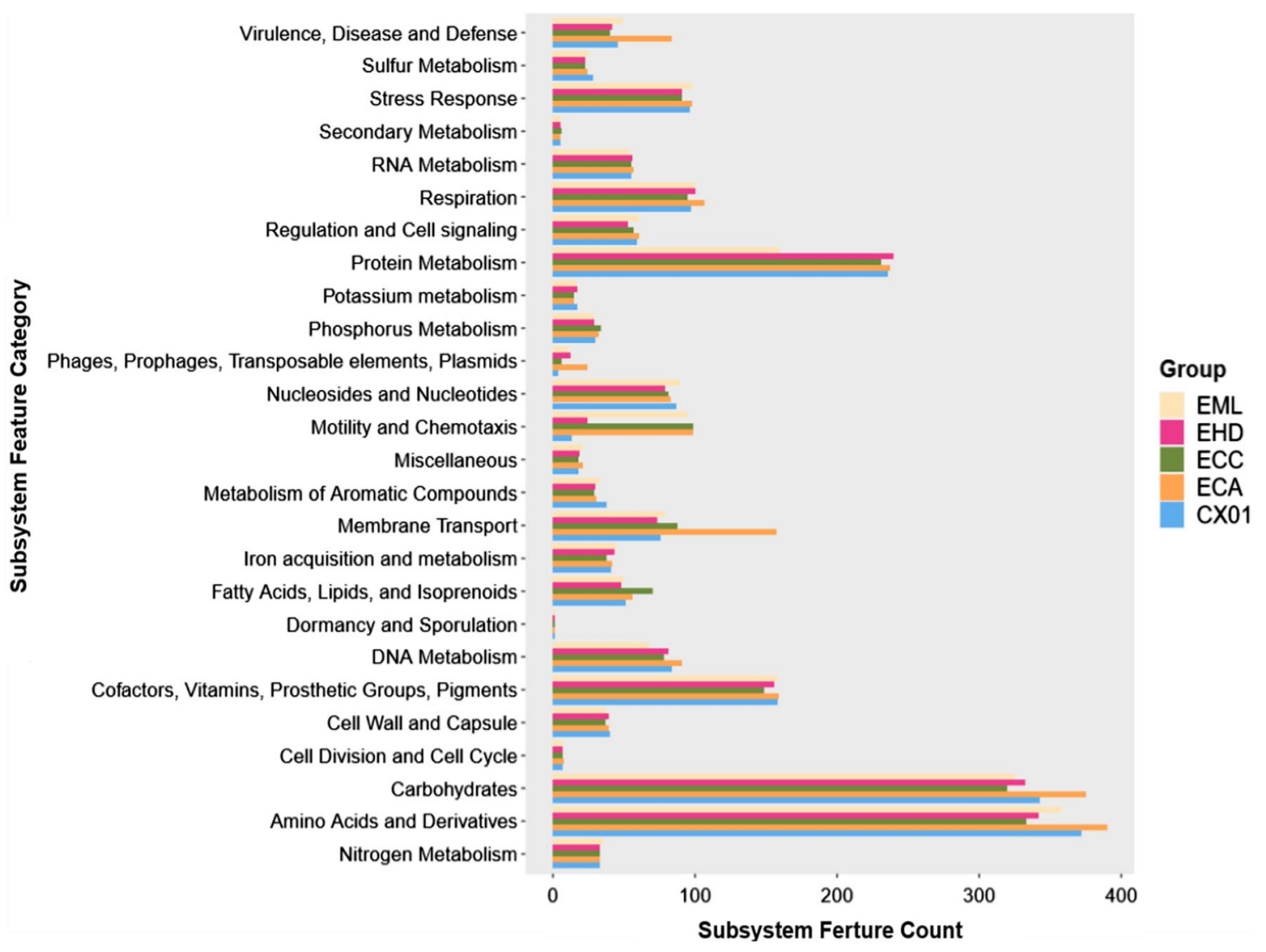

Fig. 5 Comparison of subsystem features among Enterobacter species. The data are derived from genome information of E. mori LMG 25706 (EML), E. mori CX01 (CX01, CP055276), E. cloacae ATCC 13047 (ECA, NC_014121.1), E. hormaechei DSM 16691 (EHD, NZ_CP017179.1) and E. cancerogenus CR-Eb1 (ECC, NZ_CP025225.1)

coregulated protein) and VgrG (valine glycine repeat) are present in E. mori CX01, which are secreted proteins of T6SS (Zheng and Leung 2007). Flagella play critical roles in infection processes such as motility which allows bacterium to move through the soil matrix and inside the plant, as well as in adhesion, biofilm formation, secretion of effector molecules, penetration through tissue structures to entry into eukaryotic cells (Chaban et al. 2015). We found that E. mori CX01 codes for flagellar genes (Additional file 2: Table S7), which may contribute to the bacterial pathogenesis. The Sec and Tat secretion pathways are highly conserved in bacteria, which were also identified in E. mori CX01.

As a phytopathogen, E. mori CX01 genome codes for virulence effectors, such as $\operatorname{srfBC}$ (EM_4592-4593), which are homolog proteins of Salmonella enterica involved in host colonization (Worley et al. 2000). Other putative virulence factors include VirK (EM_0966, EM_4235) whose homologue in Agrobaterium tumefaciens is associated with type IV secretion (Kalogeraki and Winans 1998), and MviM (EM_0755) which is putative oxidoreductase, and a predicted virulence factor (EM_4594) which is a homologue of SsrAB-activated protein. Pectin is a structural polysaccharide that is integral for the stability of plant cell walls. Pectinesterase (EM_1118) and pectin degradation protein (EM_3329) were identified in E. mori CX01.

Two-component systems (TCSs) mediate environmental signals to cellular responses signaling transduction commonly found in bacteria, archaea and a few eukaryotes (Stock et al. 2000). In E. mori CX01, 26 HKs (3 of which are hybrid kinases) and 31 RRs were identified (Additional file 2: Table S8). The homologs of YpdA/ YpdB system in Escherichia coli contributes to nutrient scavenging before entry into stationary phase (Fried et al. 2013). QseB/QseC system is conserved in many bacterial species, and functions as a global regulator of virulence associated with quorum-sensing signaling cascade (Weigel and Demuth 2016). GlrR/GlrK up-regulates transcription of the $\operatorname{glm} Y / g \operatorname{lm} Z$ sRNA to maintain normal synthesis of the cell wall and outer membrane when cells enter the stationary growth phase (Goepel et al. 
2011). CitA/CitB system may modulate the expression of the downstream gene cluster (citCDEFXG of CX01) in response to citrate, and $\mathrm{Cit} A / \mathrm{CitB}$ presents in one regulatory cascade with DcuS/DcuR system which induces the expression of genes for fumarate respiration in E. coli (Scheu et al. 2012). In addition, a high number of TCSs have been evolved and integrated into diverse cellular signaling circuits, such as nutrient up-taking, response to metal ions or osmotic stress, which confer the bacteria with different traits that enhance their fitness for invading host plants.

Nutrient acquisition is important for bacterial habitation in plant tissues. Consistently, a large number of transporters are present in E. mori CX01 that may allow the exchange of various substrates. There are more than 200 genes coding for $\mathrm{ABC}$ transporters that may be involved in the uptake of metals, osmoprotectant, sugar, amino acids, peptides, polyamines, vitamins, phosphate, sulphate, nitrate/nitrite, urea and quorum sensing autoinducer-2 (AI-2) in E. mori CX01 (Additional file 2: Table S9). E. mori CX01 also has the potential to incorporate a wide range of sugars through PTS systems (encoded by 53 genes) and MFS transporters (encoded by 59 genes) (Additional file 2: Tables S10, 11). In addition, bacteria have evolved strategies to compete for iron that is required for bacterial growth and full virulence of pathogens (Payne 1988). The iron uptake system includes specific iron uptake transporters, siderophores for iron chelating and siderophore receptors for utilizing heterologous siderophores. E. mori CX01 contains two ferrous iron uptake transporters, FeoABC (EM_2744-2746) and EfeBUO (EM_0794-0796), one ferrous-ion efflux pump FieF (EM_2504), two afuABC ferric-iron ABC transporters (EM_1356-1358, EM_1528-1530), fepABCDEG ferric enterobactin transporter (EM_1269, EM_1261, EM_1265, EM_1263, EM_3328, EM_1264), fhuBCD ferric hydroxamate transporter (EM_1655-1657). SitABCD (EM_1271-1274) and MntH (EM_3750) are manganese importer, and ZupT (EM_3116) zinc transporter. Ferritin family of iron storage proteins are present in all types of cells from higher eukaryotes to bacteria for dealing with the dual problem of iron availability and toxicity (Bradley et al. 2014). E. mori CX01 encodes bacterioferrin (EM_2810) and two ferrin-like protein (EM_4163, EM_4168) as iron-storage proteins. TonB-dependent siderophore receptor (EM_4081) is also present in the genome. Siderophore receptors are important components for bacterial virulence (Payne 1988), the homologue protein ferric-enterobactin (iron-bound siderophore) of E. mori (EM_1269) is associated with siderophore receptor in Enterobacter.

Many plant-associated bacteria affect plant growth by the production of phytohormones. For example, bacteria enhance plant growth by the synthesis of the plant auxin indole acetic acid (IAA), from tryptophan via three alternative pathways (Taghavi et al. 2009). E. mori CX01 encodes indolpyruvate decarboxylase IpdC (EM_3753) and the amine oxidase (EM_0329), which may be involved in two IAA synthesis pathways and the intermediate molecules may be the source for IAA biosynthesis in plant. The acetolactate decarboxylase (EM_1299) and 2,3-butanediol dehydrogenase (EM_1297) may be responsible for catalyzing the production of acetoin and 2,3-butanediol. E. mori CX01 contains two isochorismate synthase (ICS) homolog genes (EM_3834 and EM_1206), which are key enzymes for synthesis of salicylic acid (SA). Polyamines are widely distributed in bacteria, plants and animals, and play important roles in cell proliferation, tissue growth and differentiation (Tabor and Tabor 1984). Accordingly, in the genome of E. mori CX01, there are genes coding for arginine decarboxylase (SpeA, EM_3221), agmatinase (SpeB, EM_3222) ornithine decarboxylase (SpeC, EM_1172, EM_3187), S-adenosylmethionine decarboxylase (SpeD, EM_1685) and spermidine synthase (SpeE, EM_1684), which may allow the synthesis of polyamines of putrescine and spermidine.

In E. mori CX01, a variety of enzymes and regulators help bacteria to survive upon oxidative stress, including three superoxide dismutases (EM_0495, EM_0506, EM_2499), two catalases KatE (EM_0597) and KatG (EM_2529), four hydroperoxide reductases (EM_1232, EM_1233, EM_1486, EM_1836), two non-heme chloroperoxidases (EM_866, EM_1898), two thiol peroxidases (EM_3702, EM_4473), two glutathione peroxidases (EM_0311, EM_0577), glutathione reductase (EM_2640), five glutathione S-transferases (EM_0385, EM_0482, EM_1035, EM_2125, EM_3798), and three $\gamma$-glutamyl transpeptidase (EM_0837, EM_1565, EM_2716).

\section{Potential nutrient depletion ability between E. mori CX01 and Psa}

Nutrient depletion is a mode of bacterial competition, E. mori CX01 may have the potential of metabolizing a wide range of carbon substrates based on KEGG analysis, and the coding gene number is greater than that of Psa (Fig. 6). A total of 153 proteins was present in E. mori CX01, which are involved in pentose and glucuronate interconversions, fructose and mannose metabolism, galactose metabolism, starch and sucrose metabolism, amino sugar and nucleotide sugar metabolism, and C5-branched dibasic acid metabolism (Additional file 2: Table S12). The analysis also revealed 48 additional genes of the phosphotransferase system (PTS) specifically enriched in E. mori CX01 compared to Psa (Additional file 2: Table S13). E. mori CX01 encodes 30 additional biofilm formation genes compared with $P s a$ (Additional 


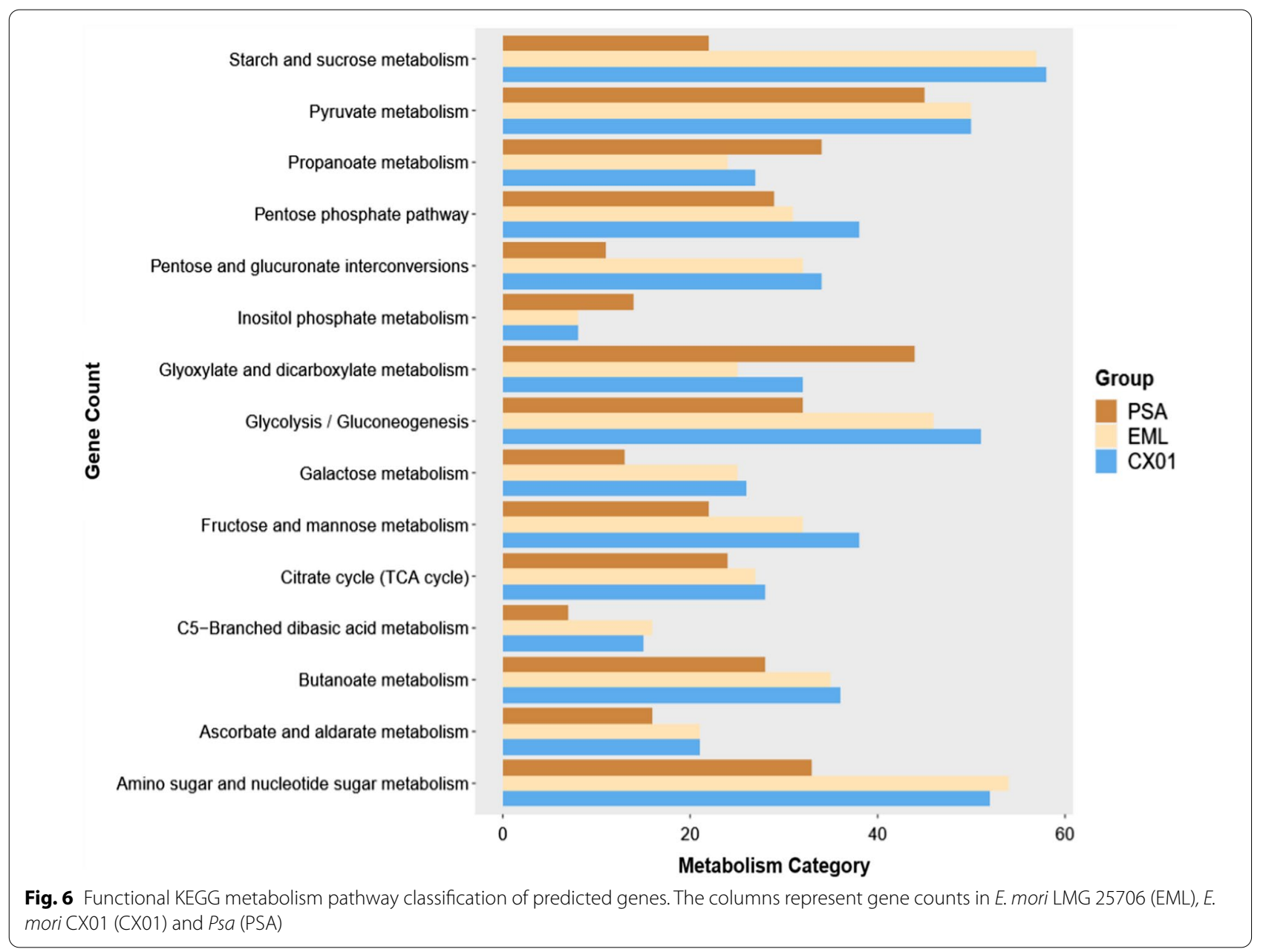

file 2: Table S14). These genes include six cyclic diguanosine monophosphate (c-di-GMP) metabolism genes, four flagellar-related genes, seven polysaccharide biosynthesis/export protein genes, one operon for curli production, eight two-component system genes and transcriptional regulator genes. In the peptidase and inhibitor activity category, 90 genes of E. mori CX01 and 58 genes of Psa were classified into this KEGG pathway. E. mori CX01 contains a more diverse set of proteases compared with $P s a$, including out membrane protease, endopeptidase, L,D-transpeptidase, dipeptidase, aminopeptidase, and metalloprotease (Additional file 2: Table S15). An unique gene in E. mori CX01 encodes leader peptidase HopD (prepilin peptidase) (EM_2811). HopD homolog in Legionella pneumophila can cleave the leader sequences from prepilin-like proteins that are required for type II protein secretion, and are indispensable for the virulence of the pathogen (Liles et al. 1999).

E. mori CX01 and Psa possess 635 and 546 transporter genes respectively. The extremely high number of transporter genes in E. mori CX01 indicates it may translocate more diverse types of substrates than Psa. The additional transporters carried by E. mori CX01 include ATP-binding cassette subfamily $B$ and C proteins, PTS and MFS transporters, as well as transport systems for nitrate/ nitrite, thiamine, spermidine/putrescine, maltose/maltodextrin, arabinogalactan oligomer/maltooligosaccharide, maltose/maltodextrin, iron, manganese, amino acids and peptides (Additional file 2: Table S16). E. mori CX01 may utilize a variety of transport machines to easily access nutrient and survive in different environments.

The number of transcriptional regulators in E. mori CX01 (151) is higher than that of Psa (103), respectively. The additional members of transcriptional regulator in $E$. mori CX01 include AraC, LysR, LacI, GntR, TetR/AcrR, LuxR, MarR, MerR, IclR, Rrf2, XRE, DtxR, TrpR, SgrR, and FrmR/RcnR family transcriptional regulators (activator or repressor) (Additional file 2: Table S17), which may contribute to substrates utilization, stress responses, and multidrug efflux system, etc. 


\section{Discussion}

In this study, a new bacteria pathogen E. mori led to kiwifruit bacterial disease in Cangxi, Sichuan province of China. The whole genome of the isolate E. mori CX01 reveals its adaptative features to survive in plant niches (Additional file 3 and 4). E. mori CX01 as a pathogen to achieve the goal of host invasion, needs to initially disarm plant basal defense networks and subsequently to liberate nutrients required for its own multiplication. We found that is represented by the metabolic potential of E. mori CX01, such as secretion systems, putative virulence effectors, two-component systems, nutrient uptake systems, phytohormone synthesis and repression of host immunity.

E. mori CX01 lacks T3SS that is considered as a prerequisite for bacterial active virulent life style in many plant pathogens (Buettner and He 2009). E. mori can easily enter into the internal tissues of its host through wounds, so T3SS may not be nessesary for its invasion. Other secretion systems including type I, II, VI secretion system, which are believed to be also related to the bacterial virulence (Green and Mecsas 2016), are coded by E. mori CX01. Besides that, the flagellar protein export apparatus are harbored by E. mori CX01, which may also exert the function of secretion of virulence factors as many different pathogens, such as Salmonella, Shigella, Serratia, and Yersinia (Macnab 2003; Soutourina and Bertin 2003; Young et al. 1999). We noted that several putative virulence factors such as $s r f B C$, VirK, MviM and pectinesterase were predicted in E. mori CX01, which may be secreted by above mentioned secretion systems and involve in the bacterial virulence.

In addition, bacterial pathogens need quickly adapt to changing environments during host entry or in a harsh environmental condition. This may be achieved by multiple signal response systems, such as two-component systems, to govern correct spatiotemporal gene expression in in E. mori CX01. A large number of two-component system genes are highly conserved within the genus Enterobacter (Capra and Laub 2012), indicating that the important role of two-component system in Enterobacter species. E. mori $\mathrm{CX} 01$ has the potential of producing volatile organic compounds acetoin and 2,3-butanediol (by acetolactate decarboxylase and 2,3-butanediol dehydrogenase), which are known to be required for full virulence in Pectobacterium carotovorum and increase the production of phenazine pyocyanin in Pseudomonas aeruginosa (Audrain et al. 2015). By contrast, some bacterial strains of plant growth-promoting rhizobacteria emitted 2,3-butanediol to induce systemic resistance in Arabidopsis thaliana (Ryu et al. 2004). Sucrose as the abundant nutrient can induce acetoin and 2,3-butanediol production in Enterobacter sp. 638 (Taghavi et al. 2009).
In plant-microbe recognition, the ability of scavenging iron is also required for bacterial progression of disease symptoms on the host plants (Franza and Expert 2013). E. mori CX01 possesses iron uptake systems that may improve its competitiveness of iron to benefit the fitness on host plants.

Though the pathogenesis mechanism of Enterobacter remains to be fully elucidated, our data may provide an insight into potential processes related to bacterial virulence. In the future, it is necessary to pay efforts on linking bacterial virulence and underlying genetic determinants, which can pave the way for understanding of serious disease development by novel pathogens.

\section{Supplementary Information}

The online version contains supplementary material available at https://doi. org/10.1186/s13568-021-01290-w.

Additional file 1: Figure S1. The CRISPR region prediction by CRISPRCasFinder. E.mori CX01 contains two putative CRISPR repeat consensus sequences at evidence level4.

Additional file 2: Table S1A. Accession numbers of gene sequences used in the MLSA analaysis. Table S1B. Accession numbers of genomes used in the MLSA analaysis. Table S2. A set of 111 COGs specifically belongs to E. mori CX01 and LMG 25706. Table S3. Specific Cluster of Orthologous Groups in Enterobacter mori CX01. Table S4. Specific Cluster of Orthologous Groups in Enterobacter mori LMG 25706. Table S5. Comparison of carbohydrate metabolism pathway in E. mori CX01 and LMG 25706 and Psa. Table S6. Subsystem Feature Category counts in Enterobacter mori strains. Table S7. Flagellar genes. Table S8. Two-component systems of histidine kinase in E. mori CX01. Table S9. ABC transporters in E. mori CX01. Table S10. PTS systems in E. mori CX01. Table S11. MFS transporters in E. mori CX01. Table S12. Carbohydrate metabolism. Table S13. Phosphotransferase system (PTS) [PATH:ko02060]. Table S14. Biofilm formation-Escherichia coli [PATH:ko02026]. Table S15. Peptidases and inhibitors [BR:ko01002]. Table S16. Transporters [BR:ko02000]. Table S17. Transcription factors [BR:ko03000].

Additional file 3. Genome annotation of Enterobacter mori CX01.

Additional file 4. Nucleic acid and protein sequences derived from Enterobacter mori CX01 open reading frames.

\section{Acknowledgements}

We are very grateful to the editors and the anonymous reviewers for their helpful comments and suggestions.

\section{Authors' contributions}

YJ, HH and RF conceived the project and designed the experiments. MZ, JW, $\mathrm{BR}, \mathrm{MX}$ and $\mathrm{GL}$ isolated the bacterial strain and performed the pathogenicity experiments. $Y Z, X H, Y L$ and $Y Y$ carried out the bioinformatics analyses. $Y J$ and $\mathrm{HH}$ wrote the manuscript. All authors read and approved the final manuscript.

\section{Funding}

This work was supported by the National Key Research and Development Plan (No. 2016YFD0100600) and the National Natural Science Foundation of China (No. 31972233), and Sichuan science and technology support project (No. 2019YFSY0025).

Availability of data and materials

All data generated or analyzed are included in this article and its additional files. For further information, please contact the author. 


\section{Code availability}

Not applicable.

\section{Declarations}

\section{Ethics approval and consent to participate}

This article does not contain any studies with human participants or animals performed by any of the authors of this investigation. The participant in the photo (Fig. 1) has obtained the informed consent and agrees to the use of the photograph.

\section{Consent for publication}

The work described has not been published before, and is not under consideration for publication elsewhere.

\section{Competing interests}

The authors declare no competing interests.

\section{Author details}

${ }^{1}$ State Key Laboratory of Plant Genomics, Institute of Microbiology, Chinese Academy of Sciences, Beijing 100101, China. ${ }^{2}$ National Plant Gene Research Center, Beijing 100101, China. ${ }^{3}$ School of Advanced Agriculture Sciences and School of Life Sciences, State Key Laboratory of Protein and Plant Gene Research, Peking University, Beijing 100871, China. ${ }^{4}$ College of Life Sciences, University of the Chinese Academy of Sciences, Beijing 100049, China. ${ }^{5}$ Cangxi Xingke Modern Agricultural Science and Technology Research Institute Co. Ltd., Cangxi 628400, China. ${ }^{6}$ Guangxi Key Laboratory of Medicinal Resource Protection and Genetic Improvement, Guangxi Botanical Garden of Medicinal Plant, Nanning 530023, China.

Received: 27 Auqust 2021 Accepted: 3 September 2021 Published online: 17 September 2021

\section{References}

Audrain B, Farag MA, Ryu CM, Ghigo JM (2015) Role of bacterial volatile compounds in bacterial biology. FEMS Microbiol Rev 39:222-233. https://doi. org/10.1093/femsre/fuu013

Bradley JM, Moore GR, Le Brun NE (2014) Mechanisms of iron mineralization in ferritins: one size does not fit all. J Biol Inorg Chem 19:775-785. https:// doi.org/10.1007/s00775-014-1136-3

Brettin T, Davis JJ, Disz T, Edwards RA, Gerdes S, Olsen GJ, Olson R, Overbeek R, Parrello B, Pusch GD, Shukla M, Thomason JA, Stevens R, Vonstein V, Wattam AR, Xia FF (2015) RASTtk: a modular and extensible implementation of the RAST algorithm for building custom annotation pipelines and annotating batches of genomes. Sci Rep 5:8365. https://doi.org/10.1038/ srep08365

Buettner D, He SY (2009) Type III protein secretion in plant pathogenic bacteria. Plant Physiol 150:1656-1664. https://doi.org/10.1104/pp.109.139089

Cao P, Li C, Tan K, Liu C, Xu X, Zhang S, Wang X, Zhao J, Xiang W (2020) Characterization, phylogenetic analyses, and pathogenicity of Enterobacter cloacae on rice seedlings in Heilongjiang province, China. Plant Dis 104:1601-1609. https://doi.org/10.1094/PDIS-12-19-2557-RE

Capra EJ, Laub MT (2012) Evolution of two-component signal transduction systems. Annu Rev Microbiol 66(1):325-347. https://doi.org/10.1146/ annurev-micro-092611-150039

Chaban B, Hughes HV, Beeby M (2015) The flagellum in bacterial pathogens: for motility and a whole lot more. Semin Cell Dev Biol 46:91-103. https:// doi.org/10.1016/j.semcdb.2015.10.032

Chikhi R, Medvedev P (2014) Informed and automated k-mer size selection for genome assembly. Bioinformatics 30(1):31-37. https://doi.org/10.1093/ bioinformatics/btt310

Darling ACE, Mau B, Blattner FR, Perna NT (2004) Mauve: multiple alignment of conserved genomic sequence with rearrangements. Genome Res 14:1394-1403. https://doi.org/10.1101/gr.2289704

de Vrind J, de Groot A, Brouwers GJ, Tommassen J, de Vrind-de JE (2003) Identification of a novel Gsp-related pathway required for secretion of the manganese-oxidizing factor of Pseudomonas putida strain GB-1. Mol Microbiol 47:993-1006. https://doi.org/10.1046/j.1365-2958.2003.03339.x
Donati I, Buriani G, Cellini A, Mauri S, Costa G, Spinelli F (2014) New insights on the bacterial canker of kiwifruit (Pseudomonas syringae pv. actinidiae). J Berry Res 4:53-67. https://doi.org/10.3233/JBR-140073

Edwards U, Rogall T, Blockerl H, Emde M, Bottger CE (1989) Isolation and direct complete nucleotide determination of entire genes. Characterization of a gene coding for 165 ribosomal RNA. Nucleic Acids Res 17:7843-7853. https://doi.org/10.1093/nar/17.19.7843

Franza T, Expert D (2013) Role of iron homeostasis in the virulence of phytopathogenic bacteria: an 'à la carte' menu. Mol Plant Pathol 14(4):429_ 438. https://doi.org/10.1111/mpp.12007

Fried L, Behr S, Jung K (2013) Identification of a target gene and activating stimulus for the YpdA/YpdB histidine kinase/response regulator system in Escherichia coli. J Bacteriol 195:807-815. https://doi.org/10.1128/JB. 02051-12

Garcia-Gonzalez T, Karina Saenz-Hidalgo H, Victoria Silva-Rojas H, MoralesNieto C, Vancheva T, Koebnik R, Dolores Avila-Quezada G (2018) Enterobacter cloacae, an emerging plant-pathogenic bacterium affecting chili pepper seedlings. Plant Pathol J 34:1-10. https://doi.org/10.5423/ppj.Oa. 06.2017 .0128

Goepel Y, Luettmann D, Heroven AK, Reichenbach B, Dersch P, Goerke B (2011) Common and divergent features in transcriptional control of the homologous small RNAs GImY and GImZ in Enterobacteriaceae. Nucleic Acids Res 39:1294-1309. https://doi.org/10.1093/nar/gkq986

Green ER, Mecsas J (2016) Bacterial secretion systems: An Overview. Microbiol Spectr 4(1). https://doi.org/10.1128/microbiolspec.VMBF-0012-2015

Hormaeche E, Edwards PR (1960) Aproposed genus Enterobacter. Int J Syst Evol Micr 10(2):71-74. https://doi.org/10.1099/0096266x-10-2-71

Kalogeraki VS, Winans SC (1998) Wound-released chemical signals may elicit multiple responses from an Agrobacterium tumefaciens strain containing an octopine-type Ti plasmid. J Bacteriol 180:5660-5667. https://doi.org/ 10.1128/jb.180.21.5660-5667.1998

Kanehisa M, Goto S (2000) KEGG: kyoto encyclopedia of genes and genomes. Nucleic Acids Res 28:27-30. https://doi.org/10.1093/nar/28.1.27

King EO, Ward MK, Raney DE (1954) Two simple media for the demonstration of pyocyanin and fluorescin. J Lab Clin Med 44(2):301-307

Koren S, Walenz BP, Berlin K, Miller JR, Bergman NH, Phillippy AM (2017) Canu: scalable and accurate long-read assembly via adaptive k-mer weighting and repeat separation. Genome Res 27(5):722-736. https://doi.org/10. $1101 /$ gr.215087.116

Kumar S, Stecher G, Tamura K (2016) MEGA7: molecular evolutionary genetics analysis version 7.0 forbigger datasets. Mol Biol Evol 33(7):1870-1874. https://doi.org/10.1093/molbev/msw054

Lane DJ (1991) 16S/23S rRNA Sequencing. In: Stackebrandt E and Goodfellow M(eds) Nucleic acid techniques in bacterial systematic, Wiley, New York, pp 115-175

Liles MR, Edelstein PH, Cianciotto NP (1999) The prepilin peptidase is required for protein secretion by and the virulence of the intracellular pathogen Legionella pneumophila. Mol Microbiol 31:959-970. https://doi.org/10. 1046/j.1365-2958.1999.01239.x

Lowe TM, Eddy SR (1997) tRNAscan-SE: a program for improved detection of transfer RNA genes in genomic sequence. Nucleic Acids Res 25:955-964. https://doi.org/10.1093/nar/25.5.955

Macnab RM (2003) How bacteria assemble flagella. Annu Rev Microbiol 57(1):77-100. https://doi.org/10.1146/annurev.micro.57.030502.090832

Majeed A, Muhammad Z, Ahmad H (2018) Plant growth promoting bacteria: role in soil improvement, abiotic and biotic stress management of crops. Plant Cell Rep 37:1599-1609. https://doi.org/10.1007/s00299-018-2341-2

McGinnis S, Madden TL (2004) BLAST: at the core of a powerful and diverse set of sequence analysis tools. Nucleic Acids Res 32:W20-W25. https://doi. org/10.1093/nar/gkh435

Mezzatesta ML, Gona F, Stefani S (2012) Enterobacter cloacae complex: clinical impact and emerging antibiotic resistance. Future Microbiol 7:887-902. https://doi.org/10.2217/fmb.12.61

Moriya Y, Itoh M, Okuda S, Yoshizawa AC, Kanehisa M (2007) KAAS: an automatic genome annotation and pathway reconstruction server. Nucleic Acids Res 35:W182-W185. https://doi.org/10.1093/nar/gkm321

Murray MG, Thompson WF (1980) Rapid isolation of high molecular weight plant DNA. Nucleic Acids Res 8:4321-4325. https://doi.org/10.1093/nar/8. 19.4321

Nagrale DT, Gawande SP, Hiremani NS, Gokte-Narkhedkar N (2020) Occurrence and pathogenicity of Enterobacter sp. causing sprout decay and 
seedling stunting of upland cotton (Gossypium hirsutum L.). J Phytopathol 168:391-398. https://doi.org/10.1111/jph.12903

Payne SM (1988) Iron and virulence in the family Enterobacteriaceae. Crit Rev Microbiol 16:81-111. https://doi.org/10.3109/10408418809104468

Ryu CM, Farag MA, Hu CH, Reddy MS, Kloepper JW, Pare PW (2004) Bacterial volatiles induce systemic resistance in Arabidopsis. Plant Physiol 134(3):1017-1026. https://doi.org/10.1104/pp.103.026583

Scheu PD, Witan J, Rauschmeier M, Graf S, Liao YF, Ebert-Jung A, Basche T, Erker W, Undena G (2012) CitA/CitB two-component system regulating citrate fermentation in Escherichia coli and its relation to the DcuS/DcuR system in vivo. J Bacteriol 194:636-645. https://doi.org/10.1128/jb.06345-11

Schroeder BK, Waters TD, du Toit LJ (2010) Evaluation of onion cultivars for resistance to Enterobacter cloacae in storage. Plant Dis 94:236-243. https://doi.org/10.1094/PDIS-94-2-0236

Soutourina OA, Bertin PN (2003) Regulation cascade of flagellar expression in Gram-negative bacteria. FEMS Microbiol Rev 27(4):505-523. https://doi. org/10.1016/s0168-6445(03)00064-0

Stackebrandt E, Ebers J (2006) Taxonomic parameters revisited: Tarnished gold standards. Microbiol Today 33:152-155

Stock AM, Robinson VL, Goudreau PN (2000) Two-component signal transduction. Ann Rev Biochem 69:183-215. https://doi.org/10.1146/annurev. biochem.69.1.183

Tabor CW, Tabor H (1984) Polyamines. Ann Rev Biochem 53:749-790. https:// doi.org/10.1146/annurev.bi.53.070184.003533

Taghavi S, Garafola C, Monchy S, Newman L, Hoffman A, Weyens N, Barac T, Vangronsveld J, van der Lelie D (2009) Genome survey and characterization of endophytic bacteria exhibiting a beneficial effect on growth and development of poplar trees. Appl Environ Microbiol 75:748-757. https:// doi.org/10.1128/aem.02239-08

Takikawa Y, Serizawa S, Ichikawa T, Tsuyumu S, Goto M (1989) Pseudomonas syringae pv. actinidiae pv. nov: the causal bacterium of canker of kiwifruit in Japan. Ann Phytopath Soc Japan 55:437-444. https://doi.org/10.3186/ jjphytopath.55.437

Weigel WA, Demuth DR (2016) QseBC, a two-component bacterial adrenergic receptor and global regulator of virulence in Enterobacteriaceae and Pasteurellaceae. Mol Oral Microbiol 31:379-397. https://doi.org/10.1111/ omi.12138
Worley MJ, Ching KHL, Heffron F (2000) Salmonella SsrB activates a global regulon of horizontally acquired genes. Mol Microbiol 36:749-761. https://doi.org/10.1046/j.1365-2958.2000.01902.x

Xiao CL, Chen Y, Xie SQ, Chen KN, Wang Y, Han Y, Luo F, Xie Z (2017) MECAT: fast mapping, error correction, and de novo assembly for single-molecule sequencing reads. Nat Methods 14(11):1072-1074. https://doi.org/10. 1038/nmeth.4432

Xin XF, Kvitko B, He SY (2018) Pseudomonas syringae: what it takes to be a pathogen. Nat Rev Microbiol 16:316-328. https://doi.org/10.1038/nrmic ro.2018.17

Xu L, Dong Z, Fang L, Luo Y, Wei Z, Guo H, Zhang G, Gu YQ, Coleman-Derr D, Xia Q, Wang Y (2019) OrthoVenn2: a web server for whole-genome comparison and annotation of orthologous clusters across multiple species. Nucleic Acids Res 47:W52-W58. https://doi.org/10.1093/nar/gkz333

Zheng J, Leung KY (2007) Dissection of a type VI secretion system in Edwardsiella tarda. Mol Microbiol 66:1 192-1206. https://doi.org/10.1111/j.13652958.2007.05993.x

Wang ZS, Tang XF, Liu SJ (1992) Identification of the pathogenic bacterium for bacterial canker on actinidia in Sichuan. J Southwest Agric Univ 6:500-503

Young GM, Schmiel DH, Miller VL (1999) A new pathway for the secretion of virulence factors by bacteria: The flagellar export apparatus functions as a protein-secretion system. Proc Natl Acad Sci U S A 96:6456-6461. https:// doi.org/10.1073/pnas.96.11.6456

Zhu B, Lou MM, Xie GL, Wang GF, Zhou Q, Wang F, Fang Y, Su T, Li B, Duan YP (2011) Enterobacter mori sp. nov., associated with bacterial wilt on Morus alba L. Int J Syst Evol Microbiol 61:2769-2774. https://doi.org/10.1099/ ijs.0.028613-0

\section{Publisher's Note}

Springer Nature remains neutral with regard to jurisdictional claims in published maps and institutional affiliations.

\section{Submit your manuscript to a SpringerOpen ${ }^{\odot}$ journal and benefit from:}

- Convenient online submission

- Rigorous peer review

- Open access: articles freely available online

- High visibility within the field

- Retaining the copyright to your article

Submit your next manuscript at $\boldsymbol{\nabla}$ springeropen.com 